\title{
OPTIMAL PLACEMENT OF PILES IN REAL GRILLAGES: EXPERIMENTAL COMPARISON OF OPTIMIZATION ALGORITHMS
}

\author{
Rimantas Belevičius*, Sergèjus Ivanikovas ${ }^{* *}$, Dmitrij Šešok ${ }^{* * * * *}$, \\ Saulius Valentinavičius", Julius Žilinskas ${ }^{* * * * * *}$ \\ *Vilnius Gediminas Technical University, Vilnius, Lithuania \\ ${ }^{* *}$ Vilnius Pedagogical University, Vilnius, Lithuania \\ *** Institute of Mathematics and Informatics, Vilnius University, Akademijos 4, Vilnius, Lithuania \\ e-mail:julius.zilinskas@mii.vu.lt,zilinskasjulius@gmail.com \\ crossref http://dx.doi.org/10.5755/j01.itc.40.2.427
}

\begin{abstract}
The aim of the article is to choose algorithms suited for optimal placement of piles in real grillages by performing experimental comparison of different global optimization algorithms. The comparison includes several algorithms: random search, metaheuristics (simulated annealing and genetic algorithm) and local optimization combined with random search. The algorithms are compared using the results of optimization of pile placement schemes of 10 practical grillages of small-to-medium scale with data obtained from several design bureaus. The best results have been achieved with simulated annealing and nonlinear optimization algorithm NEWUOA combined with heuristic random search.
\end{abstract}

Keywords: global optimization, metaheuristics, optimal design of grillage-type foundations.

\section{Introduction}

Finding a global optimum in industrial optimization problems usually is a difficult and time-consuming task. A number of studies are published comparing various optimization algorithms. A frequent pitfall with comparative studies of this type is a difficulty to generalize the results to other problems. Each search method has its own characteristics which make the various search techniques more suited to different problem domains. Eriksson and Arora (2002) presented a comparison of global optimization algorithms applied to industrial ride comfort optimization problem. The genetic algorithm (GA) was found more efficient than the simulated annealing (SA) algorithm for this particular problem. Dumas et al. (2005) studied the drag reduction in the automotive industry problem. Various modifications of GAs were compared; in the most successful case the computational time of a GA is reduced by a factor up to 7 . Fowler et al. (2008) compare the derivative-free optimization methods for groundwater supply problems. Horne and MacBeth (1998) applied global optimization algorithms to the problem of geophysical inversion and did not find pronounced advances of Tabu Search, GA and SA for this problem.

In this paper we shall concentrate on one specific class of optimization problems in civil engineering optimal design of grillage-type foundations, which are called "grillages" throughout this paper. Grillages are the most popular and effective scheme of foundations, especially in the case of weak grounds. The grillages consist of supporting piles and connecting beams. Bowles (1996), Reese et al. (2005) outline exhaustive technical details on the grillages. However, only a few works so far deal with optimization of foundation schemes. Chan et al. (2009) combine the sizing and topology optimization, however the piles are aggregated to special groups. Kim et al. (2001) minimize the differential settlements of piled rafts, again, by a special way minimizing the number of design variables. We are trying to obtain the globally minimal price of pile foundations treating all piles as a separate design variables. Belevičius and Valentinavičius (2001) and Belevičius et al. (2002) introduced the idealizations of real grillages, which are taken in the present mathematical model as well.

The optimal grillage should meet twofold criteria: the number of piles should be minimal, and connecting beams should receive minimal possible torques. In fact, here we encounter two separate optimization problems: search for the minimal number of piles and search for the minimal volume of beams. Both problems can be integrated into one with a compromise objective function. We assume that the characteristics of piles and connecting beams are given and consider the first optimization problem.

Initial data for the grillage optimization problem are the following: 
- The geometrical scheme of connecting beams;

- Cross-section data of all beams (area, moments of inertia);

- Material data of all beams (material in one beam is treated as isotropic);

- Positions of immovable piles (if any);

- Maximum allowable reactive force at any pile;

- Minimum possible distance between adjacent piles;

- Vertical and two rotational stiffnesses (along the beam and normal to the beam) of pile;

- Loading data. Active forces can be applied in the form of concentrated loads and moments at any point on beam, or in the form of distributed trapezoidal loadings at any segment of beam.

The results of optimization are the number of required piles and their positions. To solve this problem it is necessary to find such a placement of the given number of piles that reactive forces do not exceed the carrying capacities of piles. If such a placement is not possible, the number of piles should be increased.

Therefore we formulate a problem of placement of piles searching for appropriate pile positions under connecting beams. In an ideal grillage, reactive forces at all piles are identical. Practically this is hardly feasible, particularly when a designer introduces the so-called "immovable supports" that have to retain their positions and cannot change them during optimization process. Some technological constraints may also make the ideal scheme non-achievable, for example, the distance between adjacent piles cannot be too small due to the specific capacities of a pile driver. In the present work we do not consider the immovable supports and allow for a pile to take whatever position in the grillage, thus typically the piles are not placed at the joints of grillage. This fact confines the pile placement problem scope to a lowrise buildings without significant overturning moments due to horizontal thrust, e.g. due to earthquake loading or wind loads.

The objective function for minimization can be formulated in several alternative forms, e.g. the maximal vertical reactive force at a pile, the difference between the maximal and minimal reactive forces in the whole grillage, or the maximal difference between the reactive force and carrying capacity of a pile. A grillage supported by piles with different characterristics can be optimized using the third objective function mentioned. We assume that the characteristics of all piles are equal and in this case the first and last objective functions are equivalent. We use the first objective function in the current paper.

An even distribution of reactive forces or differrences between reactions and carrying capacities among all piles indicates an ideal grillage. Therefore the ideal value of the objective function or the lower bound for the minimum is known in advance. This makes the problems attractive as test sets for experimental investigation of optimization algorithms since it can be estimated how far the best value found is from the ideal one.

Our experience shows that the objective function for practical grillage optimization problems possesses many local minima points. Another complicated trait of the problem is that usually the objective function is very sensitive to the positions of piles: sometimes even a small alteration of one position leads to a significant change of the value of the objective function. All this makes the placement of piles in practical grillage a difficult global optimization problem.

In our previous work we tried to approach the problem as "black-box" global optimization by covering methods (Čiegis et al. 2006; Žilinskas 2008), genetic algorithms (Belevičius and Šešok 2008), and local optimization combined with random search (Ivanikovas et al. 2009), but the results were not inspiring even for problems with 10 and 15 piles. One possible reason is that heuristic information on the problem is not employed in the case of "black-box" optimization. In this paper we compare different strategies for solution of the problem: starting from random search and heuristic random search, then progressing to metaheuristics (simulated annealing and genetic algorithms) and local optimization combined with heuristic random search. Promising results have been achieved when heuristic random search is combined with metaheuristics and local optimization.

The algorithms are compared using the results of optimization of pile placement schemes of 10 practical grillages. All these grillages are of small-to-medium scale, requiring from 18 to 55 piles. Data for these problems are obtained from several Dutch design bureaus (courtesy of Consultancy W.F.O. B.V., Paauw B.V. Aannemingsbedrijf, Aannemingsbedrijf V. Dijk, Bouwtectuur West Friesland, Stabo Bouw B.V., Aannemingsbedrijf A. Tuin Den Helder and others) which use the professional software for structural engineering package MatrixFrame (http://www.matrix-software.com/uk/structuralengineering/matrixframe/ index.html). It is intended for an analysis and design of steel and concrete erections. Apropos, MatrixFrame implemented our software for optimization of pile placement schemes employing local search methods (Belevičius and Valentinavičius 2001). However, the current optimization routine of MatrixFrame was not capable to yield even a rational scheme of pile placement for the problems considered in this paper.

\section{Mathematical formulation}

The optimization problem is formulated as follows:

$$
f^{*}=\min _{\mathrm{x} \in \mathrm{D}} f(\mathrm{x}),
$$

where $f(\mathrm{x})$ is a nonlinear objective function of continuous variables $f: \mathfrak{R}^{n} \rightarrow \mathfrak{R}, \mathrm{x}$ represent design parameters defining positions of piles, their number is denoted by $n, \mathrm{D} \subset \mathfrak{R}^{n}$ is a feasible region. Besides of 
the global minimum $f^{*}$, one or all global minimizers $\mathrm{x}^{*}: f\left(\mathrm{x}^{*}\right)=f^{*}$ should be found. No assumptions on unimodality are included into formulation of the problem - many local minima may exist.

In this paper the maximal vertical reactive force at a pile is considered as the objective function:

$$
f(\mathrm{x})=\max _{i=1, \ldots, N_{p}} R_{i}(\mathrm{x}),
$$

where $N_{p}$ is the number of piles, $R_{i}(\mathrm{x})$ is the reactive force at the $i$-th pile.

Since a supporting pile may reside only under connecting beams, there are evident restrictions on the positions of piles: during the optimization process the piles can move only along the connecting beams. Therefore, a two-dimensional beam structure of the grillage is "unfolded" to a one-dimensional construct, and the piles are allowed to range through this space freely.

Unfortunately in such a formulation small variation of the design variable may correspond to a finite variation of the position of the pile in the physical space, what leads to discontinuity of the problem. One possibility to overcome this is to use multilevel optimization where the upper level combinatorial problem assigns piles to beams, while the lower level continuous problems aim to position the piles in the assigned beams. Another possibility is to divide search space avoiding jumps of piles from one beam to another and perform searches in such separate spaces. However both possibilities are applicable only when a considerable number of objective function evaluations can be performed. Since evaluation of objective function of pile placement based on finite element analysis is computationally expensive and we will perform only 5000 evaluations in each run of optimization algorithm, we do not think these possibilities are applicable. Unfortunately, we do not know a parameterrization that would not introduce discontinuities and lead to simpler optimization problem.

One design parameter corresponds to a position of one pile in the one-dimensional construct. The backward transformation restores the positions of piles in the two-dimensional beam structure of the grillage. The constraints for the design parameters are as follows:

$$
0 \leq x_{i} \leq L, \quad i=1, \ldots, N_{p},
$$

where $x_{i}$ is a design parameter defining the position of the $i$-th pile, $L$ is the total length of all beams in the grillage. If the minimal possible distance $\delta$ between adjacent piles is specified, there are additional constraints

$$
\left\|\mathrm{x}_{i}-\mathrm{x}_{j}\right\| \geq \delta, i \neq j,
$$

where $\mathrm{x}_{i}$ are two-dimensional coordinates of piles and $\left\|\mathrm{x}_{i}-\mathrm{x}_{j}\right\|$ denotes the distance between piles. To cope with this constraint, a penalty is included in the objective function.

A finite element program is used as a "black-box" routine to the optimization program for solution of direct problem to find reactive forces in the grillage. In the direct problem that is solved via finite element analysis, the connecting beams in the grillage are idealized as the beam elements, while the piles are treated as supports, i.e. finite element mesh nodes with given elastic boundary conditions. Since time of optimization crucially depends on time of solution the direct problem, fast problem-oriented original FORTRAN programs with a special mesh preprocessor have been developed and used.

The beam elements have 2 nodes with 6 degrees of freedom each (3 displacements along the coordinate axes and 3 rotations about these axes). The stiffness matrix for element can be found in many textbooks, e.g. by Spyrakos and Raftoyiannis (1997):

$$
\begin{aligned}
& {[K]=\left[\begin{array}{ll}
{\left[K_{11}\right]} & {\left[K_{12}\right]} \\
{\left[K_{12}\right]^{T}} & {\left[K_{22}\right]}
\end{array}\right],} \\
& {\left[K_{11}\right]=\left[\begin{array}{cccccc}
\frac{\mathrm{EA}}{\mathrm{L}} & 0 & 0 & 0 & 0 & 0 \\
0 & \frac{12 \mathrm{EI}_{\mathrm{z}}}{\mathrm{L}^{3}} & & \frac{12 \mathrm{EI}_{\mathrm{y}}}{\mathrm{L}^{3}} & -\frac{6 \mathrm{EI}_{\mathrm{y}}}{\mathrm{L}^{2}} & \\
0 & & \frac{\mathrm{GJ}}{\mathrm{L}} & \\
0 & & \frac{6 \mathrm{EI}_{\mathrm{y}}}{\mathrm{L}^{2}} & \frac{4 \mathrm{EI}_{\mathrm{y}}}{\mathrm{L}} & \\
0 & & & \frac{4 \mathrm{EI}_{\mathrm{z}}}{\mathrm{L}}
\end{array}\right] \text {, }} \\
& {\left[K_{12}\right]=\left[\begin{array}{cccccc}
-\frac{\mathrm{EA}}{\mathrm{L}} & 0 & 0 & 0 & 0 & 0 \\
0 & -\frac{12 \mathrm{EI}_{\mathrm{z}}}{\mathrm{L}^{3}} & & & & \frac{6 \mathrm{EI}_{\mathrm{z}}}{\mathrm{L}^{2}} \\
0 & & -\frac{12 \mathrm{EI}_{\mathrm{y}}}{\mathrm{L}^{3}} & -\frac{6 \mathrm{EI}_{\mathrm{y}}}{\mathrm{L}^{2}} & \\
0 & & & -\frac{\mathrm{GJ}}{\mathrm{L}} & & \\
0 & & \frac{6 \mathrm{EI}_{\mathrm{y}}}{\mathrm{L}^{2}} & \frac{2 \mathrm{EI}_{\mathrm{y}}}{\mathrm{L}} & \\
0 & -\frac{6 \mathrm{EI}_{\mathrm{z}}}{\mathrm{L}^{2}} & & & & \frac{2 \mathrm{EI}_{\mathrm{z}}}{\mathrm{L}}
\end{array}\right] \text {, }}
\end{aligned}
$$

where $E$ is the Young's modulus, $A$ is a cross-sectional area of the beam, $J, I_{z}$ and $I_{y}$ are the inertial moments of cross-section. Sub-matrix $\left[K_{22}\right]$ coincides with $\left[K_{11}\right]$ but non-diagonal members are with opposite signs. The main statics equation is

$$
[K]^{a}\{u\}^{a}=\{F\}^{a},
$$

where $a$ stands for the ensemble of elements (not shown in eq. below), $\{u\}$ are the nodal displacements, and $\{F\}$ are the active forces. The reactive forces at piles are available after obtaining the nodal displacements:

$$
R_{i}=\sum_{j}\left[K_{i j}\right] u_{j} \cdot
$$

Sensitivity analysis may be used if optimization algorithm requires information about derivatives. 


\section{Optimization algorithms}

Several algorithms have been employed for placement of piles minimizing the maximal vertical reactive force at a pile:

- Random search (RS);

- Modified random search (MRS);

- Simulated annealing (SA);

- Genetic algorithm (GA);

- Simplex method (SM);

- Variable metric method (VM);

- NEWUOA algorithm.

To make a fair comparison, the total number of objective function evaluations for each algorithm is the same: $N=5000$. The values for parameters of the algorithms have been chosen according to the results of exhaustive numerical investigation.

Random search is performed generating decision variables randomly with uniform distribution. $N$ independent random structures are generated and reactive forces at the piles are found using finite element analysis. The structure with the smallest maximal reactive force at a pile is considered to be the best solution found.

Heuristic information is employed in modified random search. Decision variables are generated randomly with uniform distribution, but additional constraint is set: the difference of two decision variables should be larger than $S$ which is obtained by dividing the half of the total length of all beams by the number of piles:

$$
\left|x_{i}-x_{j}\right| \geq S, i \neq j, S=\frac{L}{2 N_{p}} .
$$

The value of decision variable is regenerated randomly with uniform distribution if it is too close to a value of a previously generated decision variable. Such a heuristic modification is motivated by the fact that due to the usual distribution of loading over the grillage beams, the piles also should be spread over the whole space of grillage. Reactive forces at the piles are found for $N$ feasible random structures and one with the smallest maximal reactive force at a pile is considered to be the best solution found.

Simulated annealing (Kirkpatrick et al. 1983) replaces the current solution by a random solution with a probability that depends on the difference of the function values and a temperature parameter. In the beginning the temperature parameter is large allowing non-improving changes. Gradually temperature is decreased and the search becomes descent. In our implementation the initial solution is obtained using RS or MRS taking the best feasible solution out of $N$ init $=200$. Then SA algorithm is employed. A new potential set of values of decision variables is found adding independent random numbers uniformly distributed over $[-y, y]$ to the current values. The value of $y$ is gradually reduced during the solution process: in the iterations from 201 to $(2 N / 5)$ it is equal to 0.5 , in the succeeding $(2 N / 5)$ iterations -0.1 , and in the final $N / 5$ iterations -0.05 . All these values are chosen on the basis of numerical experiments. If the value of the objective function with the set of new values of decision variables is better than the previous one, the values of decision variables values are modified with probability 1 ; otherwise - with probability

$$
e^{\frac{\Delta h}{t_{1} / \ln \left(1+t_{2} \cdot j\right)}},
$$

where $\Delta h$ denotes the difference between the best so far value of the objective function and its value after modification, $t_{1}$ is the initial temperature, $t_{2}$ is the annealing speed, and $j$ denotes the iteration number. The SA algorithm proceeds until $j$ exceeds the given number of iterations $N$.

Genetic algorithms (Goldberg 1989) simulate evolution (selection, mutation, crossover) in which a population of solutions evolves improving values of the objective function. In our implementation, the initial population of popsize individuals is generated using RS or MRS. The new generation is obtained from the previous one using selection, crossover and mutation operations. During the selection, popsize/2 pairs of individuals are selected for breeding by the roulette principle. The individuals with a better objective function value have higher probability to be selected. The probability that the $j$-th individual with an objective function value $f_{j}$ will be chosen is

$$
p_{j}=\frac{1}{f_{j} \sum_{i} \frac{1}{f_{i}}}
$$

where the sum operator covers entire population. The crossover between two individuals chosen for breeding is performed with probability $p_{\text {c cross }}$; the crossover position is obtained randomly from the interval of $\left[1, N_{a}-1\right]$. The mutation is performed with probability $p \_m u t$ to each decision variable: the value is augmented by a random number uniformly distributed over $[-y, y]$. The algorithm proceeds for the given number of generations $(G)$. As in SA, the value of $y$ is gradually reduced in the same manner: $y=0.5$ for the generations $2^{\text {nd }}$ to the $(2 G / 5), y=0.1$ for the generations $(2 G / 5+1)$ to $(4 G / 5)$, and $y=0.05$ for the last $G / 5$ iterations.

The simplex method by Nelder and Mead (1965) is a general method for optimization of nonlinear multidimensional function requiring only function evaluations, but not derivatives. A simplex is the geometrical figure consisting of $n+1$ vertices in $n$ dimensions. The simplex method takes a series of steps, moving the vertex of the simplex where the objective function is worst. We use implementation of the method form Press (1992). The initial simplex is constrained around the best solution obtained using RS or MRS in the given number of iterations $N$ init: the first coordinate of the first vertex is by $S / \overline{2}$ smaller than the first coordinate in the best solution, the other vertices are formed adding $S / 2$ to one of the coordinates at a time. 
The simplex method proceeds while the overall number of objective function evaluations reaches $N$.

Variable metric methods (also known as quasiNewton methods) are based on Newton's method to find the stationary point of the objective function where the gradient is 0 . However Hessian matrix does not need to be computed, but it is updated by analyzing successive gradient vectors. We use implementtation of Broyden-Fletcher-Goldfarb-Shanno (BFGS) method from Press (1992). Since variable metric method requires gradient information, the results of sensitivity analysis are used. The starting point is the best solution obtained using RS or MRS in the given number of iterations $N_{-}$init. Then local optimization is performed. Initialization and local search is repeated until the overall number of function evaluations reaches $N$. Evaluations of objective function and gradient (sensitivity analysis) function are counted, computational time of these two functions is not very different.

The NEWUOA algorithm is an iterative algorithm for nonlinear optimization. A quadratic model is used in a trust region procedure for adjusting the variables (Powell 2006). The quadratic model interpolates the function at $2 n+1$ points, only one interpolation point is altered on each iteration. The starting point is the best solution obtained using RS or MRS in the given number of iterations $N$ init. The NEWUOA algorithm is stopped when the number of objective function evaluations reaches $N$.

The values of main parameters of the algorithms are summarized in Table 1. Other parameters, for example the initial temperature for SA or probabilities of crossover and mutation for GA are tuned to the problems under consideration.

Table 1. Parameters for algorithms

\begin{tabular}{ll}
\hline Algorithm & Parameters \\
\hline RS & $N=5000$ \\
MRS & $N=5000$ \\
SA & $N \_$init $=200, N=5000, t_{1}=5, t_{2}=2$ \\
GA & popsize $=20, p \_$cross $=0.9, p \_$mut $=$ \\
& $0.3, G=N /$ popsize $=5000 / 20=250$ \\
SM & $N \_$init $=300, N=5000$ \\
VM & N_init $=300, N=5000$ \\
NEWUOA & N_init $=300, N=5000$ \\
\hline
\end{tabular}

\section{Numerical examples and timings}

The characteristics of test problems are presented in Table 2. There are no immovable piles, therefore the numbers of piles $\left(N_{p}\right)$ and decision variables $(n)$ coincide. $R_{\text {allw }}$ denotes the allowable reactive force at the piles. In all problems it is equal for all piles of grillage. The number of piles is obtained by dividing the sum of loadings by the allowable reactive force of piles - the number of piles cannot be less than this number. $R_{\text {ideal }}$ denotes the ideal theoretical value. The ideal solution is when all reactive forces at the piles are equal. In this case the reactive forces are equal to the sum of loadings divided by the number of piles. In all these problems the proportion between the total loading and the allowable reaction is such that the engineering solution requires achieving almost the ideal solution. This is the main reason, why these problems are difficult to solve. The typical grillage together with obtained pile positions is shown in Figure 1. The two-dimensional schemes of all grillages with indicated positions of piles are depicted in Figures 3 to 12 in Appendix.

Table 2. Characteristics of problems

\begin{tabular}{lcrcr}
\hline Problem No & $N_{p}$ & $L$ & $R_{\text {allw }}$ & \multicolumn{1}{c}{$R_{\text {ideal }}$} \\
\hline 1 & 25 & 172.9 & 325 & 307.47 \\
2 & 18 & 52.9 & 110 & 104.12 \\
3 & 31 & 84.1 & 105 & 101.85 \\
4 & 31 & 84.9 & 105 & 101.24 \\
5 & 30 & 63.9 & 100 & 97.51 \\
6 & 37 & 80.1 & 100 & 97.53 \\
7 & 23 & 129.1 & 300 & 287.35 \\
8 & 34 & 137.9 & 250 & 236.28 \\
9 & 17 & 97.6 & 250 & 244.71 \\
10 & 55 & 315.61 & 350 & 349.05 \\
\hline
\end{tabular}

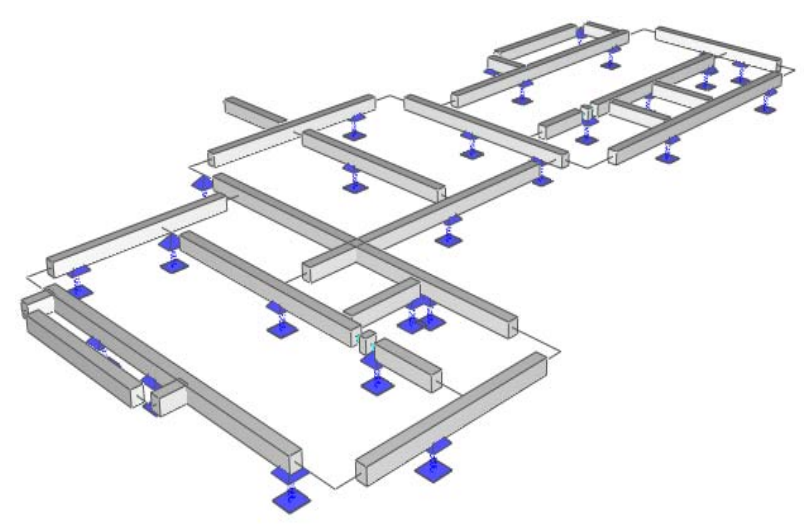

Figure 1. Grillage No 1 (according to Table 2)

The black-box finite element routine for evaluation of the objective function in a library format together with 10 grillage data (txt format) and FORTRAN example file are available at: http://soften.ktu.lt/ $\sim$ mockus/grillage/contgrillage.html.

The algorithms were tested on a personal computer with Intel(R) Xeon(R) CPU E5420@2.50GHz, 3069 MB RAM, 32-bit Operating System. 28 independent runs were performed for each algorithm. Tables 3 and 4 present the average results when RS (Table 3 ) or MRS (Table 4) is used for search and initialization. As can be seen comparing these tables, the heuristic modification significantly improves the results and enables optimal design of real grillages. When a pure random search is used for initialization, the results are much worse. In all tables the bold type highlights the best achieved results. The best average results are 
achieved by SA and NEWUOA. Results of GA are close, while RS, MRS, SM and VM perform worse. When MRS is used for initialization NEWUOA significantly outruns SA for Problem 8 (34 piles). It shows slightly better results also for Problems 4 (31) and 6 (37). Nearly in all cases the GA shows the third best solutions; it never outruns the SA or NEWUOA algorithms. The results of SM and VM are sometimes even worse than that of MRS what means that it is not worth to spend time to find local minima for these problems, but rather to search wider globally since local searches do not improve the value of the objective function significantly. Good performance of SA and GA does not surprise since their parameters (for example the initial temperature for SA or probabilities of crossover and mutation for GA) are tuned to the problems under consideration. NEWUOA optimizes a quadratic model of the objective function and performs better than local optimization of the objective function itself. The use of a model allows overcoming of problems of discontinuity and non-differentiability.
The best obtained results in 28 runs are rendered in Table 5. MRS is used for initialization. NEWUOA obtains the best solutions for two problems, for other problems SA provides better results. An ideal solution was not obtained for any problem in any of 28 independent runs; in each run the objective function was evaluated 5000 times. The best values found in 28 runs normalized to $R_{\text {ideal }}$ (discrepancies in \%) are shown in Figure 2. The last column of Table 5 presents discrepancies between the best obtained results and the corresponding ideal value. The best objective values found differ from ideal from 2.2\% (Problem 2, 18 piles) to $46.8 \%$ (Problem 8, 34 piles). As it can be expected, generally discrepancy is larger for problems with the larger number of piles. Thus, for Problem 8 (34 supports), 10 (55), and 6 (37) the discrepancies between the best obtained and ideal values are respectively $46.8 \%, 32.7 \%$, and $20.2 \%$, while for Problems 2 (18), 9 (17), and 5 (30) - 2.2\%, 3.4\%, and $3.6 \%$. Also, the character of loading distribution over the grillage plays a crucial role too.

Table 3. Average of the best values found in 28 runs when RS is used for search and initialization

\begin{tabular}{lrrrrrrr}
\hline Problem No & \multicolumn{1}{l}{$R_{\text {ideal }}$} & \multicolumn{1}{l}{ RS } & \multicolumn{1}{l}{ SA } & \multicolumn{1}{l}{ GA } & \multicolumn{1}{l}{ SM } & \multicolumn{1}{l}{ VM } & NEWUOA \\
\hline 1 & 307.47 & 593.33 & 463.08 & 490.01 & 633.77 & 547.63 & $\mathbf{4 4 0 . 3 1}$ \\
2 & 104.12 & 153.07 & 119.74 & 119.60 & 172.79 & 152.38 & $\mathbf{1 1 8 . 6 8}$ \\
3 & 101.85 & 258.45 & $\mathbf{1 3 4 . 5 9}$ & 142.24 & 274.06 & 230.73 & 139.88 \\
4 & 101.24 & 265.41 & 139.63 & 149.87 & 264.42 & 251.90 & $\mathbf{1 3 6 . 4 3}$ \\
5 & 97.51 & 318.16 & 132.52 & 142.53 & 310.40 & 290.67 & $\mathbf{1 2 8 . 7 9}$ \\
6 & 97.53 & 460.31 & $\mathbf{1 6 2 . 0 9}$ & 175.90 & 460.16 & 435.57 & 188.60 \\
7 & 287.35 & 472.74 & 372.63 & 380.48 & 507.99 & 450.81 & $\mathbf{3 6 9 . 0 3}$ \\
8 & 236.28 & 695.60 & $\mathbf{4 5 5 . 6 3}$ & 479.87 & 721.70 & 655.56 & 467.22 \\
9 & 244.71 & 402.17 & 307.55 & 321.27 & 427.50 & 385.72 & $\mathbf{2 9 6 . 2 7}$ \\
10 & 349.05 & 1321.48 & 806.46 & 890.51 & 1319.49 & 1163.32 & $\mathbf{7 7 1 . 2 6}$ \\
\hline
\end{tabular}

Table 4. Average of the best values found in 28 runs when MRS is used for search and initialization

\begin{tabular}{lrrrrrrr}
\hline Problem No & $R_{\text {ideal }}$ & MRS & SA & GA & SM & VM & NEWUOA \\
\hline 1 & 307.47 & 470.90 & $\mathbf{3 7 1 . 5 5}$ & 405.36 & 486.50 & 454.01 & 394.92 \\
2 & 104.12 & 125.71 & $\mathbf{1 0 9 . 1 0}$ & 112.49 & 131.75 & 127.64 & 113.35 \\
3 & 101.85 & 144.46 & $\mathbf{1 1 9 . 0 6}$ & 124.48 & 153.82 & 143.60 & 119.80 \\
4 & 101.24 & 141.18 & 117.10 & 123.72 & 147.46 & 139.82 & $\mathbf{1 1 6 . 8 1}$ \\
5 & 97.51 & 126.08 & $\mathbf{1 0 6 . 2 5}$ & 112.23 & 133.28 & 128.13 & 108.68 \\
6 & 97.53 & 160.18 & 132.22 & 144.16 & 172.38 & 160.85 & $\mathbf{1 3 2 . 0 7}$ \\
7 & 287.35 & 379.80 & $\mathbf{3 1 4 . 1 1}$ & 332.11 & 402.36 & 382.45 & 330.16 \\
8 & 236.28 & 494.83 & 413.57 & 444.88 & 520.98 & 491.25 & $\mathbf{3 8 5 . 9 9}$ \\
9 & 244.71 & 343.91 & $\mathbf{2 8 1 . 1 2}$ & 294.62 & 369.06 & 334.90 & 292.28 \\
10 & 349.05 & 702.53 & 562.79 & 636.87 & 759.33 & 705.57 & $\mathbf{5 5 9 . 8 8}$ \\
\hline
\end{tabular}

Average times of one run in seconds are shown in Table 6. One numerical experiment takes from approximately 1.5 (algorithm GA, Problem 2, 18 piles) to 41 minutes (SM, Problem 8, 34 piles). Peculiarly, the more complex-looking grillages 10 (55 supports) and 6 (37) take shorter time for almost all algorithms. The related question is, why in several cases RS takes longer time than, e.g., MRS or GA that employ additional computations. All this is due to the fact that the dimensions of direct finite element problem are varying: the finite element mesh is prepared automatically in view of the distribution of supports given by the optimizer, and of distribution character of the loading. Plausible, the more "smooth" loading together with more sophisticated algorithms always 
render more even distribution of supports which re-

sults in a lesser finite element mesh.

Table 5. The best values found in 28 runs

\begin{tabular}{lrlllllrr}
\hline Problem No & \multicolumn{1}{c}{$R_{\text {ideal }}$} & MRS & SA & GA & SM & VM & NEWUOA & Discrepancy, \% \\
\hline 1 & 307.47 & 430.67 & $\mathbf{3 3 9 . 3 0}$ & 360.73 & 436.68 & 429.34 & 370.79 & 10.4 \\
2 & 104.12 & 119.16 & $\mathbf{1 0 6 . 3 6}$ & 106.52 & 125.83 & 123.18 & 107.18 & 2.2 \\
3 & 101.85 & 138.37 & $\mathbf{1 0 7 . 2 5}$ & 115.04 & 138.58 & 135.54 & 109.00 & 5.3 \\
4 & 101.24 & 131.15 & $\mathbf{1 0 6 . 8 0}$ & 112.10 & 132.10 & 133.65 & 108.16 & 5.5 \\
5 & 97.51 & 116.88 & 102.00 & 104.58 & 120.42 & 120.74 & $\mathbf{1 0 1 . 0 5}$ & 3.6 \\
6 & 97.53 & 148.24 & $\mathbf{1 1 7 . 2 6}$ & 119.65 & 151.95 & 146.01 & 125.37 & 20.2 \\
7 & 287.35 & 351.71 & $\mathbf{2 9 8 . 1 1}$ & 310.24 & 355.82 & 358.73 & 306.31 & 3.7 \\
8 & 236.28 & 440.42 & 357.67 & 363.15 & 426.74 & 430.11 & $\mathbf{3 4 6 . 9 4}$ & 46.8 \\
9 & 244.71 & 318.38 & $\mathbf{2 5 3 . 0 0}$ & 274.55 & 339.68 & 313.40 & 270.68 & 3.4 \\
10 & 349.05 & 670.72 & $\mathbf{4 6 3 . 3 4}$ & 519.07 & 698.15 & 648.84 & 486.46 & 32.7 \\
\hline
\end{tabular}

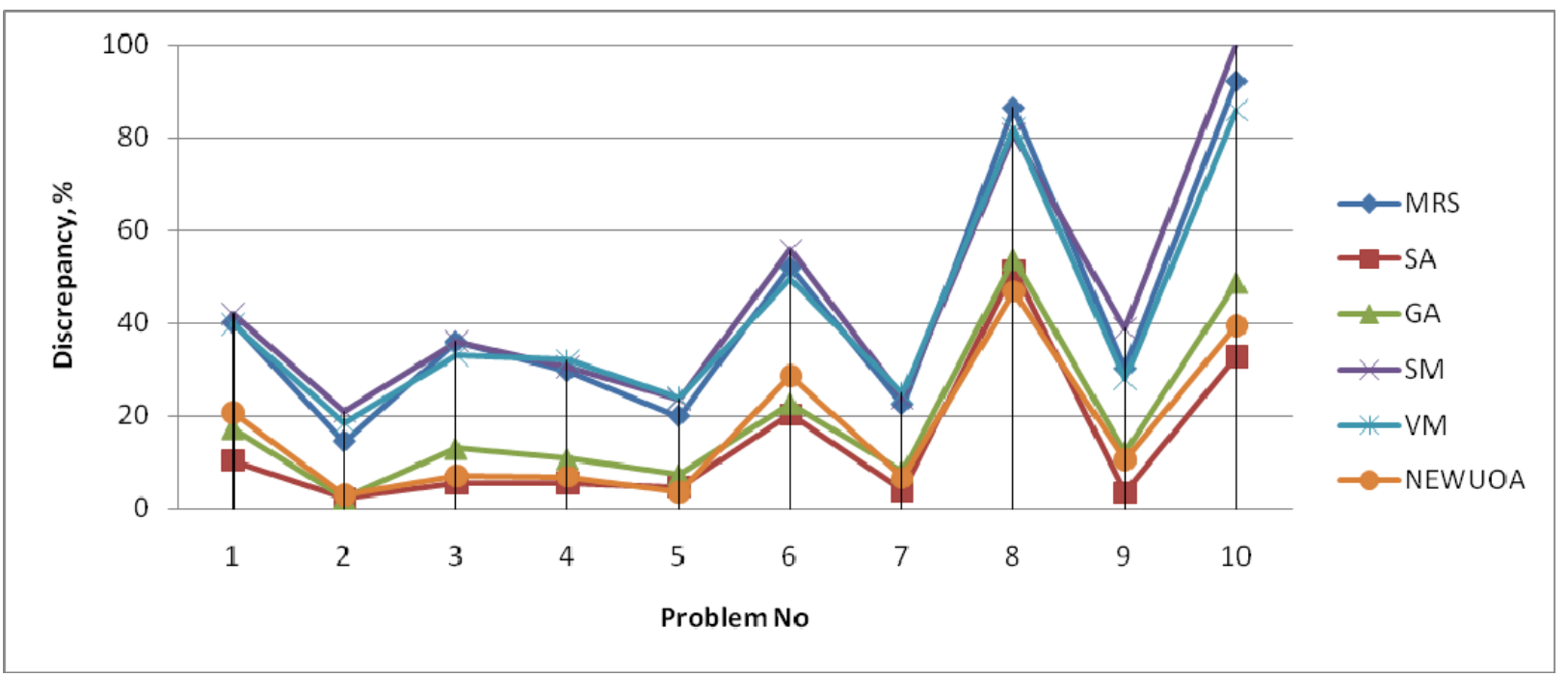

Figure 2. The best values found in 28 runs normalized to $R_{\text {ideal }}$ (discrepancies in \%)

Table 6. Average times of one run, sec

\begin{tabular}{lcrrrrrrr}
\hline Problem No & $N_{a}$ & \multicolumn{1}{c}{ RS } & MRS & \multicolumn{1}{c}{ SA } & GA & SM & VM & NEWUOA \\
\hline 1 & 25 & 899 & 943 & 960 & 844 & $\mathbf{8 2 1}$ & 855 & 878 \\
2 & 18 & 92 & 96 & 91 & $\mathbf{8 2}$ & 90 & 91 & 91 \\
3 & 31 & 835 & 876 & 822 & $\mathbf{7 3 2}$ & 811 & 801 & 753 \\
4 & 31 & 1015 & 1045 & 1046 & 935 & $\mathbf{9 1 0}$ & 914 & 916 \\
5 & 30 & 319 & 319 & 302 & $\mathbf{2 7 6}$ & 313 & 318 & 315 \\
6 & 37 & 811 & 823 & 742 & 718 & $\mathbf{6 7 4}$ & 761 & 750 \\
7 & 23 & 649 & 695 & 683 & 635 & $\mathbf{6 2 2}$ & 660 & 656 \\
8 & 34 & 3009 & 2982 & 2956 & 2668 & 2467 & $\mathbf{2 4 5 5}$ & 2489 \\
9 & 17 & 424 & 417 & 410 & $\mathbf{3 5 9}$ & 413 & 419 & 423 \\
10 & 55 & 2198 & 2136 & 2125 & 1934 & 1826 & $\mathbf{1 8 1 1}$ & 1850 \\
\hline
\end{tabular}

Comparing the timings of all algorithms, the clear winners here are the GA and SM. However, the other algorithms are not far behind, until $\sim 18 \%$ in ultimate cases. Thus, the timings cannot be treated as the decisive factor for comparison of these algorithms. This is due to the fact that most of the time is spent for evaluation of the objective function. In such cases it is worth to use optimization algorithms with more auxiliary computations, as NEWUOA is. Although auxilia- ry computations improve the results, optimization time is still quite similar.

\section{Conclusions}

Computer that is common to a typical civil engineering design bureaus and a reasonable computation time for engineering practice do not allow solution of the problem of pile placement till global optimality. However, the ideal solution usually is not required for 
the engineering purposes. The simulated annealing, genetic algorithm and nonlinear optimization algorithm NEWUOA applied from a good random point can be successfully used to obtain rational pile placement schemes under the small- and moderatescale grillages.

The results of simplex and variable metric methods show that the problems of pile placement have a number of local minima and it is not worth to spend time to find local minima for these problems, but rather to search wider globally. This is why simulated annealing and genetic algorithm perform better for these problems. NEWUOA optimizes a quadratic model of the objective function and performs better than local optimization of the objective function itself. Evaluation of objective function of pile placement based on finite element analysis is computationally expensive. Because of this, timings of all algorithms are quite similar when stopping condition is the number of function evaluations. Therefore timings cannot be treated as the decisive factor for application of the algorithms to this problem. In such cases the use of optimization algorithms with more auxiliary computations is worth as can be seen from good results of NEWUOA - auxiliary computations improve the results, but do not prolong optimization time considerably.

Several runs of the algorithm are necessary to find good solutions. Inclusion into the algorithm even some information about the problem (due to the usual distribution of loading over the grillage beams, the piles also should be spread over the whole space of grillage) significantly improves the results - this is general to all algorithms tested.

\section{Acknowledgements}

This work was supported by the Lithuanian State Science and Studies Foundation within the project B03/2007 "Global optimization of complex systems using high performance computing and grid technologies". The work of the third author was also partially supported by the Research Council of Lithuania (contract No MOS-10/2010 "Use of stochastic global optimization methods in engineering").

\section{References}

[1] R. Belevičius, D. Šešok. Global optimization of grillages using genetic algorithms. Mechanika 6(74), 2008, 38-44.

[2] R. Belevičius, S. Valentinavičius. Optimisation of grillage-type foundations. In: Proceedings of 2 nd European ECCOMAS and IACM Conference "Solids, Structures and Coupled Problems in Engineering", Cracow, Poland 26-29 June, 2001.

[3] R. Belevičius, S. Valentinavičius, E. Michnevič. Multilevel optimization of grillages. Journal of Civil Engineering and Management 8(2), 2002, 98-103

[4] J.E. Bowles. Foundation Analysis and Design. $5^{\text {th }} e d$. McGraw-Hill, 1996.

[5] C.M. Chan, L.M. Zhang, J.T.M. Ng. Optimization of pile groups using hybrid genetic algorithms. Jour- nal of Geotechnical and Geoenvironmental Engineering 135(4), 2009, 497-505.

[6] R. Čiegis, M. Baravykaitė, R. Belevičius. Parallel global optimization of foundation schemes in civil engineering. Lecture Notes in Computer Science 3732, 2006, 305-313.

[7] L. Dumas, V. Herbert, F. Muyl. Comparison of global optimization methods for drag reduction in the automotive industry. Lecture Notes in Computer Science 3483, 2005, 948-957.

[8] P. Eriksson, J.S. Arora. A comparison of global optimization algorithms applied to a ride comfort optimization problem. Structural and Multidisciplinary Optimization 24(2), 2002, 157-167.

[9] K.R. Fowler, J.P. Reese, C.E. Kees, J.E. Dennis Jr., C.T. Kelley, C.T. Miller, C. Audet, A.J. Booker, G. Couture, R.W. Darwin, M.W. Farthing, D.E. Finkel, J.M. Gablonsky, G. Gray, T.G. Kolda. Comparison of derivative-free optimization methods for groundwater supply and hydraulic capture community problems. Advances in Water Resources 31(5), 2008, 743-757.

[10] D.E. Goldberg. Genetic Algorithms in Search, Optimization and Machine Learning. Addison-Wesley, New York, 1989.

[11] S. Horne, C. MacBeth. A comparison of global optimisation methods for near-offset VSP inversion. Computers \& Geosciences 24(6), 1998, 563-572.

[12] S. Ivanikovas, E. Filatovas, J. Žilinskas. Experimental investigation of local searches for optimization of grillage-type foundations. In: Čiegis R, Henty D, Kågström B, Žilinskas J (eds) Parallel Scientific Computing and Optimization. Vol. 27 of Springer Optimization and Its Applications, Springer, 2009, 103-112. doi:10.1007/978-0-387-09707-7 9.

[13] K.N. Kim, S.-H. Lee, K.-S. Kim, C.-K. Chung, M.M. Kim, H.S. Lee. Optimal pile arrangement for minimizing differential settlements in piled raft foundations. Computers and Geotechnics 28(4), 2001, 235-253.

[14] S. Kirkpatrick, C.D.J. Gelatt, M.P. Vecchi. Optimization by simulated annealing. Science 220(4598), 1983, 671-680.

[15] J.A. Nelder, R. Mead. A simplex method for function minimization. The Computer Journal 7(4), 1965, 308313.

[16] M.J.D. Powell. The NEWUOA software for unconstrained optimization without derivatives. In: Di Pillo $G$, Roma $M$ (eds) Large-Scale Nonlinear Optimization. Vol. 83 of Nonconvex Optimization and Its Applications, Springer, 2006, 255-296.

[17] L.C. Reese, W.M. Isenhower, S.-T. Wang. Analysis and Design of Shallow and Deep Foundations. John Wiley \& Sons, 2006.

[18] C. Spyrakos, J. Raftoyiannis. Linear and Nonlinear Finite Element Analysis in Engineering Practice. Algor Publishing Division, 1997.

[19] W.H. Press. Numerical Recipes in Fortran 77: The Art of Scientific Computing. 2nd edn. Cambridge University Press, 1992.

[20] J. Žilinskas. Branch and bound with simplicial partitions for global optimization. Mathematical Modelling and Analysis 13(1), 2008, 145-159. doi:10.3846/ 1392-6292.2008.13.145-159. 


\section{Appendix: Solutions of the problems}

The two-dimensional schemes of all grillages with indicated positions of piles are depicted in Figs. 3 to 12. The values of decision variables of these solutions are given in Table 7.

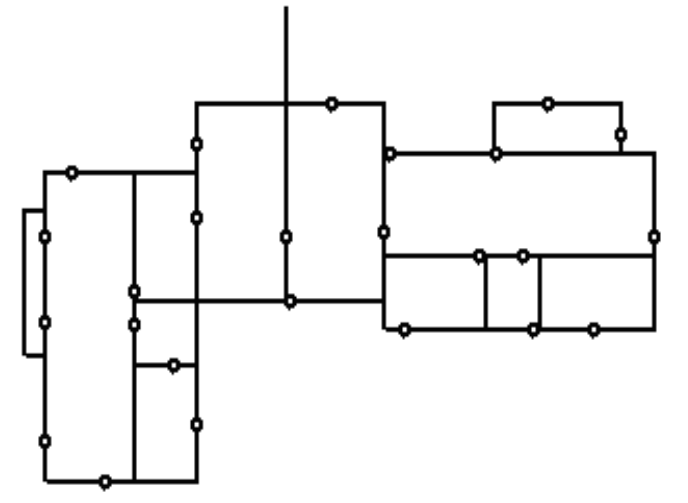

Figure 3. The best found solution of grillage No 1

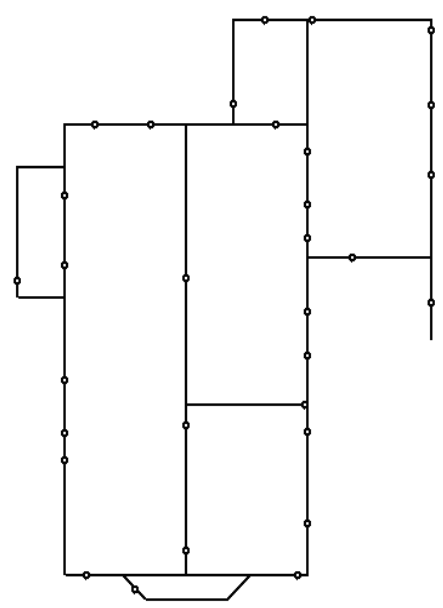

Figure 5. The best found solution of grillage No 3

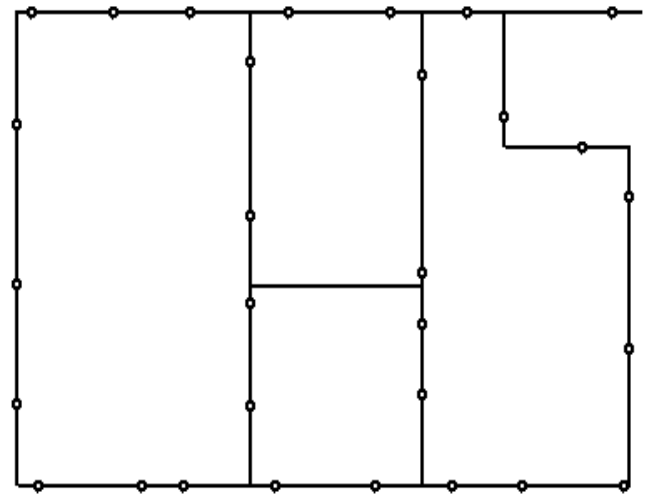

Figure 7. The best found solution of grillage No 5

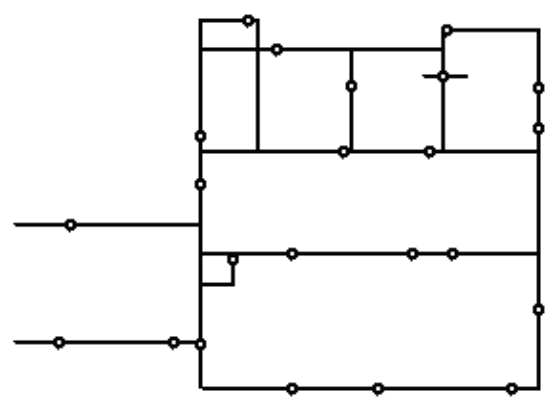

Figure 9. The best found solution of grillage No 7

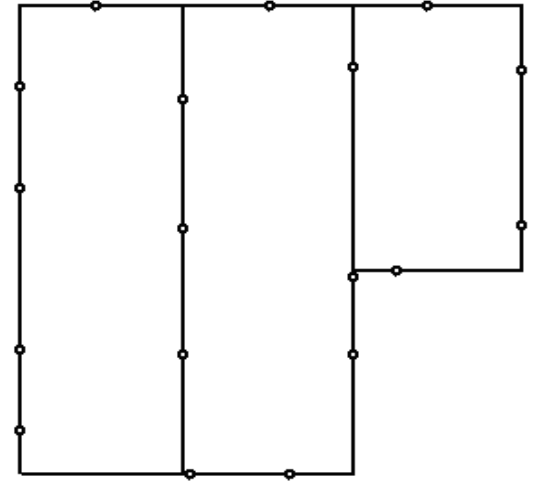

Figure 4. The best found solution of grillage No 2

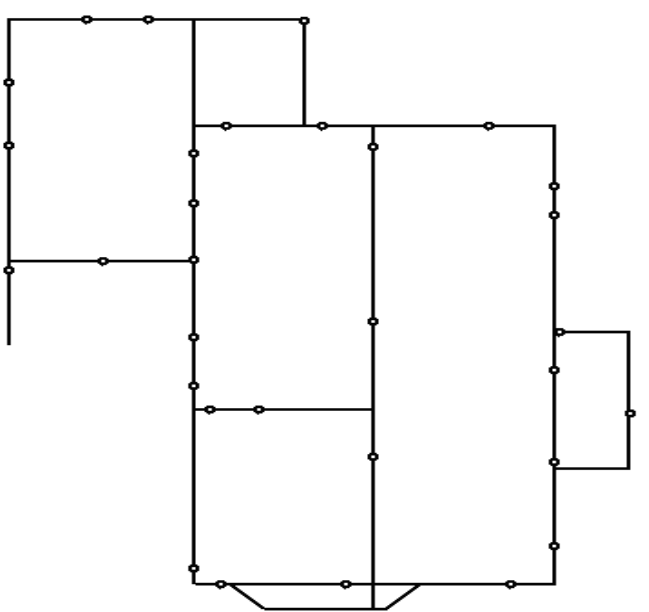

Figure 6. The best found solution of grillage No 4

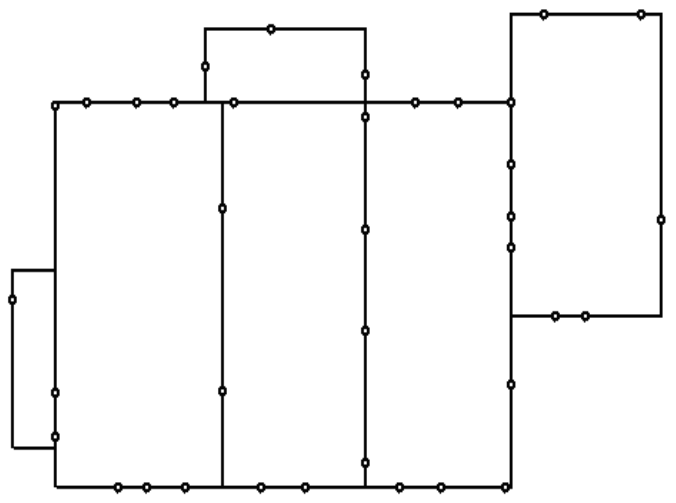

Figure 8. The best found solution of grillage No 6

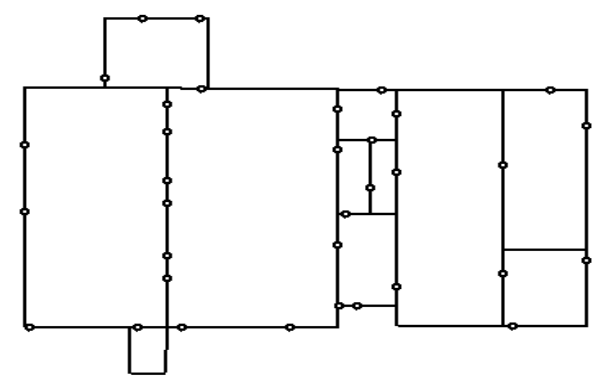

Figure 10. The best found solution of grillage No 8 


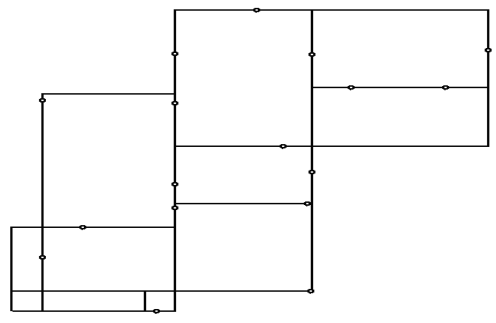

Figure 11. The best found solution of grillage No 9

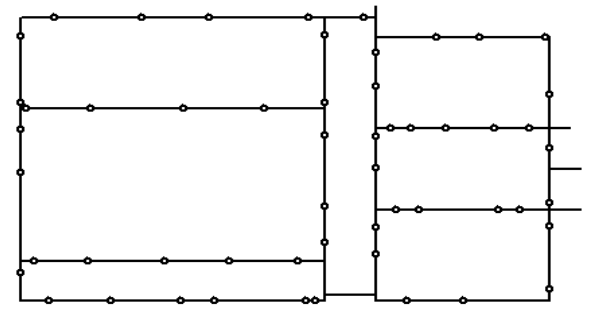

Figure 12. The best found solution of grillage No 10

Table 7. The best found solutions: values of decision variables in one-dimensional construct of grillage (with an accuracy of $1 \mathrm{~cm}$ )

\begin{tabular}{|c|c|c|c|c|c|c|c|c|c|c|}
\hline Pile No & 1 & 2 & 3 & 4 & 5 & 6 & 7 & 8 & 9 & 10 \\
\hline 1 & 7.75 & 0.88 & 0.43 & 1.92 & 1.42 & 3.05 & 3.99 & 0.20 & 4.20 & 2.68 \\
\hline 2 & 12.70 & 2.55 & 6.34 & 5.09 & 3.51 & 4.68 & 7.73 & 4.10 & 13.45 & 8.44 \\
\hline 3 & 16.28 & 5.90 & 7.05 & 6.66 & 6.33 & 5.58 & 13.53 & 5.70 & 15.58 & 14.98 \\
\hline 4 & 25.52 & 7.97 & 8.39 & 8.68 & 9.69 & 11.43 & 16.75 & 9.55 & 21.38 & 18.05 \\
\hline 5 & 26.95 & 12.10 & 11.37 & 13.30 & 11.49 & 12.22 & 21.72 & 15.42 & 33.49 & 26.58 \\
\hline 6 & 34.27 & 14.70 & 13.17 & 14.55 & 13.03 & 14.94 & 28.23 & 18.18 & 44.70 & 27.41 \\
\hline 7 & 42.97 & 17.38 & 15.66 & 16.51 & 15.71 & 18.68 & 33.48 & 18.78 & 52.09 & 31.47 \\
\hline 8 & 46.08 & 21.75 & 18.90 & 17.95 & 18.19 & 21.34 & 35.26 & 23.53 & 57.56 & 39.64 \\
\hline 9 & 50.44 & 23.34 & 22.67 & 19.22 & 19.41 & 24.05 & 41.56 & 27.24 & 64.41 & 45.85 \\
\hline 10 & 60.06 & 27.69 & 27.18 & 25.25 & 20.32 & 26.11 & 53.41 & 33.28 & 65.52 & 55.15 \\
\hline 11 & 74.54 & 29.88 & 30.70 & 28.55 & 23.78 & 28.41 & 57.20 & 40.98 & 69.26 & 60.29 \\
\hline 12 & 79.69 & 33.08 & 33.04 & 31.97 & 25.44 & 29.27 & 67.17 & 49.08 & 71.58 & 67.45 \\
\hline 13 & 86.11 & 37.54 & 35.03 & 36.39 & 29.65 & 32.29 & 74.52 & 55.71 & 77.01 & 72.34 \\
\hline 14 & 92.52 & 39.38 & 36.14 & 37.92 & 32.34 & 35.07 & 80.56 & 60.11 & 84.33 & 79.55 \\
\hline 15 & 95.57 & 41.36 & 38.07 & 40.05 & 33.66 & 35.73 & 82.92 & 65.42 & 89.64 & 85.56 \\
\hline 16 & 101.84 & 45.07 & 38.93 & 42.39 & 35.72 & 36.79 & 89.77 & 71.11 & 92.35 & 91.83 \\
\hline 17 & 104.65 & 48.25 & 40.30 & 46.33 & 36.56 & 38.04 & 91.87 & 76.71 & 97.45 & 94.96 \\
\hline 18 & 115.06 & 51.15 & 44.65 & 47.06 & 38.37 & 41.80 & 97.93 & 79.62 & & 100.98 \\
\hline 19 & 124.16 & & 47.95 & 50.02 & 40.39 & 47.48 & 106.70 & 82.07 & & 109.61 \\
\hline 20 & 126.28 & & 49.76 & 56.63 & 41.92 & 48.14 & 111.53 & 88.51 & & 117.11 \\
\hline 21 & 134.85 & & 51.71 & 58.93 & 43.31 & 49.06 & 116.94 & 93.21 & & 125.61 \\
\hline 22 & 141.23 & & 54.61 & 61.96 & 45.36 & 50.81 & 124.74 & 95.22 & & 130.89 \\
\hline 23 & 146.27 & & 56.59 & 64.22 & 50.47 & 51.85 & 126.57 & 98.59 & & 140.81 \\
\hline 24 & 160.35 & & 62.38 & 65.82 & 51.72 & 54.06 & & 99.71 & & 142.96 \\
\hline 25 & 165.41 & & 65.91 & 66.72 & 53.33 & 55.02 & & 102.29 & & 150.34 \\
\hline 26 & & & 69.84 & 70.60 & 54.86 & 56.50 & & 103.42 & & 152.28 \\
\hline 27 & & & 72.83 & 72.85 & 56.83 & 58.67 & & 105.86 & & 159.33 \\
\hline 28 & & & 74.36 & 74.64 & 58.86 & 59.37 & & 107.16 & & 161.21 \\
\hline 29 & & & 77.79 & 77.68 & 60.40 & 62.91 & & 113.67 & & 164.43 \\
\hline 30 & & & 79.54 & 80.36 & 63.29 & 64.08 & & 116.93 & & 169.04 \\
\hline 31 & & & 80.83 & 81.48 & & 64.93 & & 120.26 & & 172.18 \\
\hline 32 & & & & & & 66.32 & & 128.00 & & 181.62 \\
\hline 33 & & & & & & 70.58 & & 130.02 & & 185.62 \\
\hline 34 & & & & & & 71.56 & & 136.10 & & 191.77 \\
\hline 35 & & & & & & 74.35 & & & & 195.28 \\
\hline 36 & & & & & & 77.33 & & & & 206.05 \\
\hline 37 & & & & & & 79.57 & & & & 210.60 \\
\hline 38 & & & & & & & & & & 213.56 \\
\hline 39 & & & & & & & & & & 220.57 \\
\hline 40 & & & & & & & & & & 228.86 \\
\hline 41 & & & & & & & & & & 232.77 \\
\hline 42 & & & & & & & & & & 240.32 \\
\hline 43 & & & & & & & & & & 243.90 \\
\hline 44 & & & & & & & & & & 251.15 \\
\hline 45 & & & & & & & & & & 257.90 \\
\hline 46 & & & & & & & & & & 260.88 \\
\hline 47 & & & & & & & & & & 267.21 \\
\hline 48 & & & & & & & & & & 270.61 \\
\hline 49 & & & & & & & & & & 275.98 \\
\hline 50 & & & & & & & & & & 279.52 \\
\hline 51 & & & & & & & & & & 285.84 \\
\hline 52 & & & & & & & & & & 292.54 \\
\hline 53 & & & & & & & & & & 295.00 \\
\hline 54 & & & & & & & & & & 300.91 \\
\hline 55 & & & & & & & & & & 306.62 \\
\hline Objective & & & & & & & & & & \\
\hline $\begin{array}{l}\text { function } \\
\text { value }\end{array}$ & 339.30 & 106.36 & 107.25 & 106.80 & 101.05 & 117.26 & 298.11 & 346.94 & 253.00 & 463.34 \\
\hline Algorithm & SA & SA & SA & SA & NEWUOA & SA & SA & NEWUOA & SA & SA \\
\hline
\end{tabular}

Received November 2010. 